\title{
Molecular characterization of a Hog1-type MAPK, MlpHog1, from Melampsora larici-populina
}

\author{
Dan Yu • Ruixi Li $\cdot$ Zhongdong Yu $\cdot$ Zhimin Cao
}

Received: 18 February 2016/Accepted: 21 March 2016/Published online: 21 April 2016

(C) The Author(s) 2016. This article is published with open access at Springerlink.com

\begin{abstract}
The obligate biotrophic fungus Melampsora larici-populina (Mlp) causes devastating poplar foliar rust disease. In pathogens, the Hog 1-type mitogen-activated protein kinase (MAPK) gene is involved in both pathogenesis and the response to various stresses, including osmotic stress. However, there are few reports on its role in M. larici-populina. In the present study, we identified and characterized the Hog1-type MAPK gene, MlpHog1, in M. larici-populina. The MlpHog1 protein consists of 356 amino acids and contains a conserved TGY motif. The function of MlpHogl was probed by mutant complementation studies. Expression of MlpHogl could partially complement the osmoregulation, sexual reproduction and pathogenicity defects of the Fusarium graminearum Hogl mutant and largely rescued the response of the mutant to cytoplasm membrane and oxidative stresses. These observations suggest that MlpHog1 may play a role in the infectious growth of M. larici-populina and the response of the fungus to various environmental stresses.
\end{abstract}

Keywords Poplar foliar rust - Melampsora laricipopulina $\cdot$ Hog1-type mitogen-activated protein kinase . Function

Electronic supplementary material The online version of this article (doi:10.1007/s12600-016-0513-6) contains supplementary material, which is available to authorized users.

D. $\mathrm{Yu} \cdot \mathrm{R} . \mathrm{Li} \cdot \mathrm{Z} . \mathrm{Yu} \cdot \mathrm{Z} . \mathrm{Cao}(\square)$

College of Forestry, Northwest A\&F University, Yangling 712100

Shaanxi, China

e-mail: zmcao@nwsuaf.edu.cn

\section{Introduction}

Melampsora larici-populina (Basidiomycetes, Pucciniales) is an obligate biotrophic pathogen, similar to many other rust fungi, that has a complex heteroecious macrocyclic lifestyle. This rust completes its life cycle on two different hosts (poplar as the telial host and larch as the aecial host) and successively produces five types of spores, including pycniospores, aeciospores, urediniospores, teliospores and basidiospores (Hacquard et al. 2011; Cao et al. 2000). The poplars that are widely distributed throughout the Northern Hemisphere are commonly used in the plant science community as model trees and recently have received increasing attention as a renewable source of biomass for the energy, lumber and pulping industries (Wan et al. 2013; Duplessis et al. 2009). As the most devastating and widespread pathogen of poplars, M. larici-populina restricts the use of these trees for environmental and wood production in many parts of the world (Steenackers et al. 1996). Indeed, the distribution of foliar rust disease caused by $M$. larici-populina is widespread in China, throughout the northeast, northwest and southwest regions. Furthermore, this disease is difficult to control because the new races cause the breakdown of poplar resistance and thus limit the development of plantations (Cao et al. 1998; Tian et al. 2000). Recent advances in the genomics of poplars and M. larici-populina have helped to define new strategies to facilitate the study of this tree-rust fungus model pathosystem (Hacquard et al. 2011). 
Mitogen-activated protein kinase (MAPK) cascades function as key signal transducers that use protein phosphorylation/dephosphorylation cycles to disseminate information among evolutionarily conserved pathways (Hamel et al. 2012). In general, MAPK cascades in eukaryotes consist of three interlinked protein kinases (PKs) activated sequentially (Widmann et al. 1999) and different conserved MAPK signalling pathways are used by eukaryotic organisms to control gene expression, differentiation, cell survival, and apoptosis in response to diverse extracellular triggers (Herskowitz 1995; Treisman 1996). In budding yeast, five MAPK pathways regulate mating, invasive growth, cell wall integrity, hyperosmolarity responses, and ascospore formation (Chen and Thorner 2007). The high osmolarity glycerol (HOG) pathway is required for the accumulation of osmoprotectant molecules and the maintenance of an osmotic gradient across the stressed plasma membrane when yeast cells are grown under hypertonic conditions (Hamel et al. 2012). Numerous Hogl homologues have been identified in plant pathogenic fungi. Osm1 deletion mutants of Magnaporthe oryzae, the rice blast fungus, are highly sensitive to osmotic stress, exhibiting severe morphological defects when grown under hyperosmotic conditions (Dixon et al. 1999). The Hogl gene in the wheat scab fungus $F$. graminearum is important for regulating hyphal growth, plant infection, and hyperosmotic and general stress responses (Zheng et al. 2012), and the Hog-homologous stress-activated MAPK BcSakl in Botrytis cinerea, which causes gray mold disease, has a significant impact on pathogenesis and is involved in the response to oxidative stress (Segmuller et al. 2007). In the human pathogen Candida albicans, CaHogl is activated by various stress conditions, plays a role in cell wall biosynthesis and integrity, and is important for pathogenesis (Monge et al. 2006).

Given its obligate biotrophic status, Mlp is not amenable to genetic transformation, which has hindered the molecular investigations of this rust fungus. In this study, we cloned a Hog1-type MAPK gene named MlpHog1 and characterized its function by mutant complementation. This study provides important information for understanding the role of MlpHog1.

\section{Materials and methods}

Strains and culture conditions

The Chinese Mlp strain Wh03 was propagated on 1- to 2-year-old potted Populus purdomii plants, as described previously (Cao et al. 1998). Fresh urediniospores were harvested from the infected poplar leaves. The $F$. graminearum strains used in this study (Table 1) were maintained at $25^{\circ} \mathrm{C}$, as described previously (Hou et al. 2002). To test sensitivity to various stresses, vegetative growth was assayed on complete medium (CM) plates with $1 \mathrm{M} \mathrm{NaCl}, 0.01 \%$ SDS or $0.05 \% \mathrm{H}_{2} \mathrm{O}_{2}$ at $25^{\circ} \mathrm{C}$ for 3 days, as described previously (Wang et al. 2011, 2012).

Nucleic acid manipulations

Total RNA of urediniospores was extracted using the RNeasy Plant Mini Kit (QIAGEN, USA) following the recommended protocol. DNaseI treatment was used to remove genomic DNA. First-strand cDNA was synthesized with an Oligo $(\mathrm{dT})_{18}$ primer using the Thermo Scientific RevertAid First Strand cDNA Synthesis Kit (Thermo Fisher, USA) according to the manufacturer's instructions and used for cloning.

Isolation and sequence analysis of MlpHogl

Based on the sequence of the Mlp87970 gene in the 98AG31 genome deposited at Joint Genome Institute (JGI), MlpHog1 was cloned from the cDNA of Mlp strain Wh03 using the primers H1-F and H1-R (Table 1) and FastPfu DNA Polymerase (TransGen Biotech, China). DNA sequencing was performed by Beijing AuGCT Biological Technology. The molecular weight of the deduced protein was predicted using the ProtParam program (http://web.expasy.org/protparam/). Homologues in a set of fungal genomes were determined by Blastp searching at the Broad Institute (http://www.broadinstitute.org/) or NCBI (http://www. ncbi.nlm.nih.gov/). Multiple sequence alignments were performed using ClustalX. Phylogenetic analysis was performed using the neighbour-joining (NJ) method in MEGA6. The GenBank accession number of MlpHog1 is KT183031. 
Table 1 Primers and strains used in this study

\begin{tabular}{|c|c|c|}
\hline Primer & \multicolumn{2}{|l|}{ Sequence $\left(5^{\prime} \rightarrow 3^{\prime}\right)$} \\
\hline $\mathrm{H} 1-\mathrm{F}$ & \multicolumn{2}{|l|}{ ATGGCCGATTTTGCCAAAC } \\
\hline H1-R & \multicolumn{2}{|l|}{ TCAAGCAGCAGGGGCAGC } \\
\hline $\mathrm{H} 1-\mathrm{F} / \mathrm{RP}$ & \multicolumn{2}{|c|}{ CAGATCTTGGCTTTCGTAGGAACCCAATCTTCAATGGCCGATTTTGCCAAACTG } \\
\hline $\mathrm{H} 1-\mathrm{R} / \mathrm{RP}$ & \multicolumn{2}{|c|}{ CACCACCCCGGTGAACAGCTCCTCGCCCTTGCTCACAGCAGCAGGGGCAGCAAGAC } \\
\hline Strain & Genotype/Comment & Source \\
\hline PH-1 & A wild-type strain of Fusarium graminearum & (Cuomo et al. 2007) \\
\hline$\Delta F g H o g l$ & The hog 1 deletion mutant of PH-1 & (Zheng et al. 2012) \\
\hline $\mathrm{H}-4$ & $\Delta F g H o g 1$ complemented with RP27 promoter :: MlpHog1 & This study \\
\hline H-6 & $\Delta F g H o g 1$ complemented with RP27 promoter :: MlpHog1 & This study \\
\hline H-8 & $\Delta F g H o g 1$ complemented with RP27 promoter :: MlpHog1 & This study \\
\hline $\mathrm{H}-11$ & $\Delta F g H o g 1$ complemented with RP27 promoter :: MlpHogl & This study \\
\hline
\end{tabular}

Complementation of the F. graminearum hog1 mutant with MlpHog1

For complementation assays, the PCR product amplified with primers H1-F/RP and H1-R/RP (Table 1) was cloned into pFL2 using the yeast gap repair approach (Bourett et al. 2002). The resulting construct was transformed into protoplasts of the F. graminearum hogl mutant, as described previously (Hou et al. 2002). Geneticin-resistant transformants were confirmed by polymerase chain reaction (PCR) analysis as harbouring the plasmid.

Aerial hyphae of 7-day-old carrot agar cultures were pressed down with sterile $0.1 \%$ Tween 20 to induce sexual reproduction, as described previously (Bowden and Leslie 1999; Zheng et al. 2012). Perithecium formation and cirrhi production were examined approximately 2 weeks after induction. Freshly harvested conidia were re-suspended to a final concentration of $2 \times 10^{5}$ conidia $/ \mathrm{ml}$ in sterile distilled water (Ding et al. 2009). Flowering wheat heads of cultivar XiaoYan 22 were drop-inoculated with $10 \mu \mathrm{l}$ of conidia suspensions, as described previously (Gale et al. 2002). To maintain humidity, the inoculated wheat heads were capped with a plastic bag for $48 \mathrm{~h}$. After removing the plastic bags, the wheat plants were grown in a greenhouse. Scab symptoms were examined, and the disease index for each strain was estimated by counting the number of diseased spikelets per wheat head at 14 days postinoculation (dpi).

\section{Results}

Cloning and description of MlpHogl

The genome of $M$. larici-populina was previously sequenced (Duplessis et al. 2011), facilitating the study of genes involved in pathogen virulence. Based on sequence information of Hogl from M. larici-populina reference strain 98AG31(Protein ID 87970) (Hamel et al. 2012), an open reading frame of $1071 \mathrm{bp}$ was obtained from the Chinese Mlp strain Wh03, which is designated MlpHogl in the present study (GenBank accession number KT183031). The deduced MlpHog1 protein consists of 356 amino acids with a predicted molecular mass of 40.48 kDa. MlpHog1 contains a conserved TGY motif at positions 171 to 173 in the activation loop (Online Resource 1), which is present in the stress-activated protein kinase subgroup of MAPKs (Robinson and Cobb 1997).

MlpHog1 shares greater than $80 \%$ identity with its orthologues from other model fungi, including $S$. cerevisiae Hog1 (83\% identity), $S$. pombe Sty1 (85\% identity), M. oryzae Osm1 (88\% identity) and F. graminearum Hog1 (85\% identity) (Online Resource 1). A phylogenetic analysis revealed that $\mathrm{MlpHog} 1$ clusters with other Basidiomycete fungi and is most closely related to rust fungi (Fig. 1). 


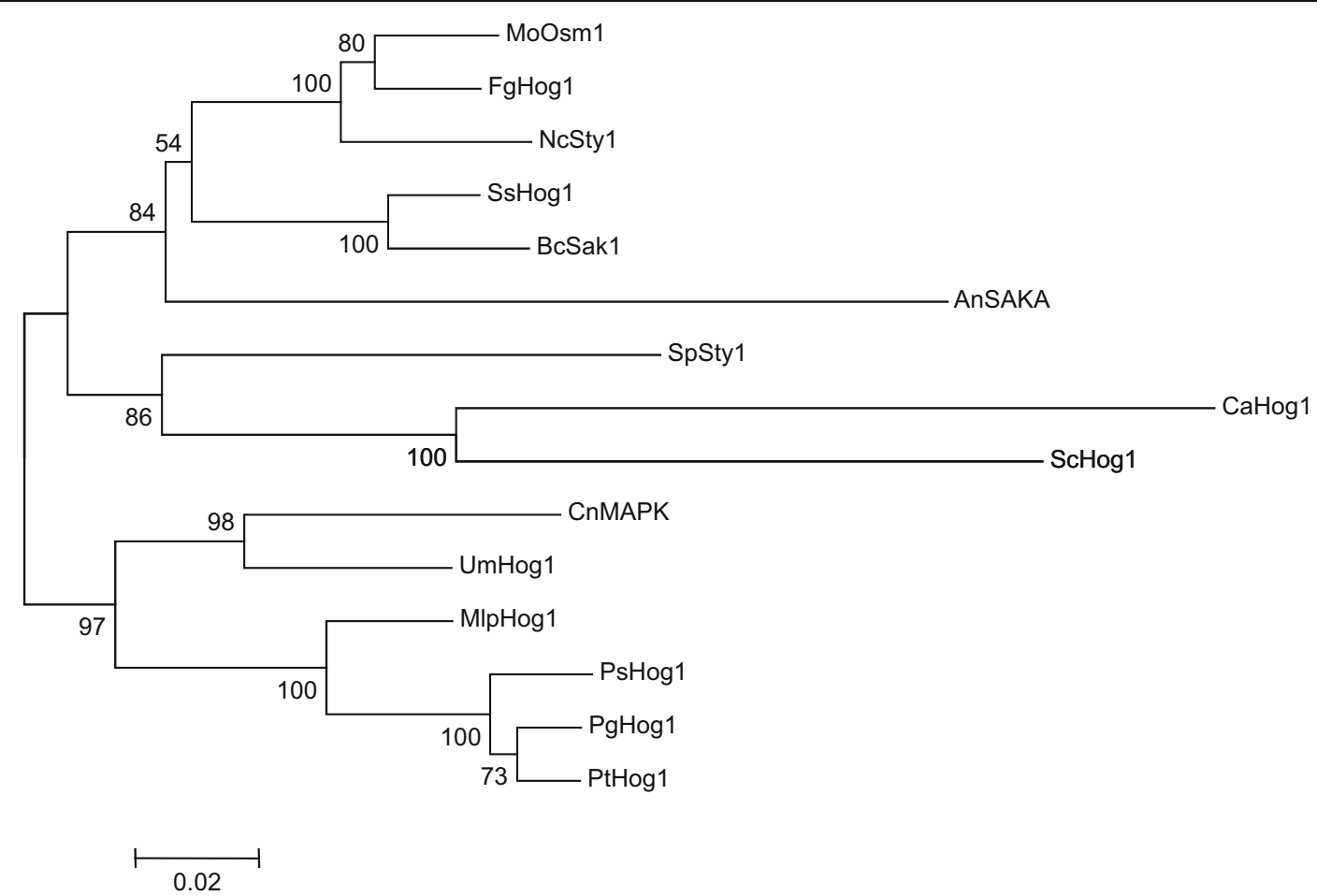

Fig. 1 Phylogenetic analysis of Hog 1 genes. Amino acid sequences encoded by $\mathrm{Hog} 1$ genes from Aspergillus nidulans (AAF97243), B. cinerea (XP001558337), C. albicans (CAWT_04100), Cryptococcus neoformans var. neoformans (XP569949), F. graminearum (FGSG_09612), M. larici-populina (KT183031), M. oryzae (AAF09475), Neurospora crassa (XP_962163), P. graminis f. sp. tritici (PGTG_04715), P. striformis f. sp. tritici (PSTG_13544), P. triticina

Complementation of defects in response to various environmental stresses in the F. graminearum Hog 1 mutant by $\mathrm{MlpHog} 1$

Given the lack of an available transformation system for $M l p$, we heterologously expressed MlpHogl in the F. graminearum Hog1 mutant to investigate its function. A construct harbouring MlpHogl driven by the RP27 promoter was transformed into protoplasts of the F. graminearum Hogl mutant (Zheng et al. 2012). Eleven resulting geneticin-resistant transformants were obtained, four of which carried the MlpHogl construct, as confirmed by PCR. The four transformants exhibited identical phenotypes, though only data regarding transformant $\mathrm{H}-4$ are provided here.

On CM plates, the wild-type $F$. graminearum strain $\mathrm{PH}-1$ produced white and aerial colonies, whereas the F. graminearum Hog1 mutant displayed less aerial hyphal growth and a reduced growth rate (Fig. 2) (Zheng
(PTTG_09272), S. cerevisiae (CAA97680), S. pombe (NP592843), Sclerotinia sclerotiorum (SS1G_07590), and Ustilago maydis (XP758504) were examined. The unrooted phylogram was constructed based on NJ analysis. The confidence of groupings was estimated by 1000 bootstrap replicates. The numbers next to the branching points indicate the percentage of replicates supporting each branch

et al. 2012). The colony of transformant H-4 was white and more aerial than the $\mathrm{FgHog} 1$ mutant, and its growth rate was significantly increased compared with that of the FgHogl mutant, which were similar to PH-1 (Fig. 2). These results indicated that expression of the MlpHogl gene in F. graminearum complemented most of the vegetative growth defects of the Hog1 mutant.

After a 3-day incubation on $\mathrm{CM}$ with $1 \mathrm{M} \mathrm{NaCl}$, the FgHogl mutant displayed no obvious growth (Fig. 2) (Zheng et al. 2012), whereas the white and aerial hyphae of transformant H-4 were visible, similar to wild-type $\mathrm{PH}-1$. However, growth of the transformant was significantly slower than that of PH-1 $(P=0.05)$ (Fig. 2), indicating partial complementation of the hyperosmotic stress response defect of the F. graminearum Hog 1 mutant by the MlpHogl gene.

Because the FgHogl mutant is involved in the response to cytoplasm membrane and oxidative stresses in F. graminearum (Zheng et al. 2012), we also evaluated 


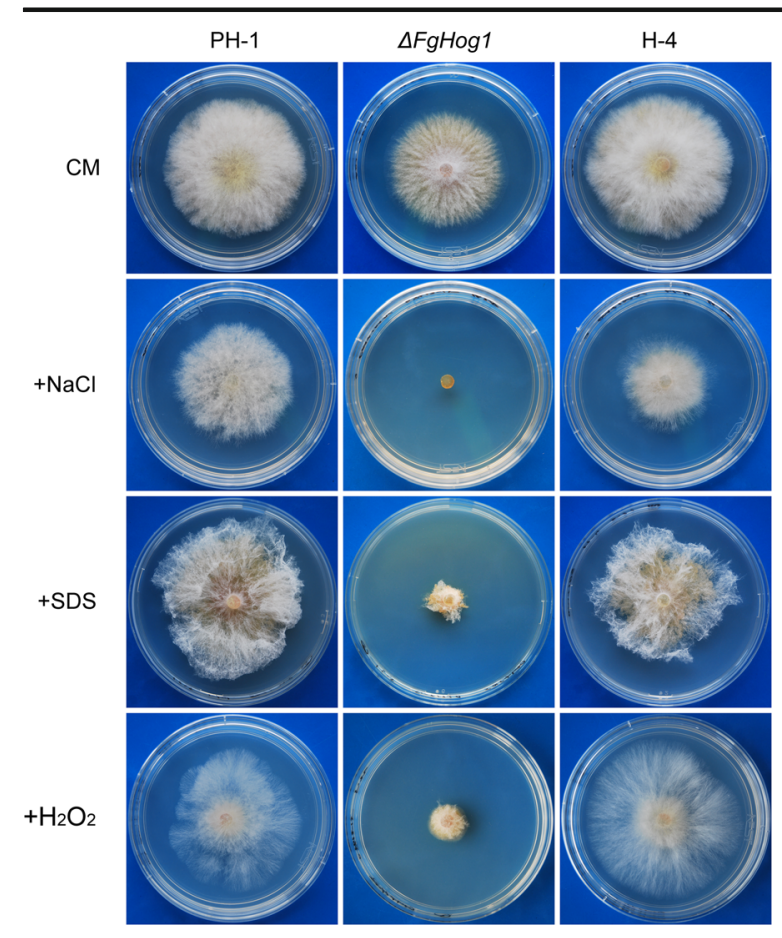

Fig. 2 Colony morphology of Fusarium graminearum strains. Colonies of the wild-type strain (PH-1), FgHogl deletion mutant, and complemented strain $(\mathrm{H}-4)$ grown on $\mathrm{CM}$ plates with $1 \mathrm{M}$ $\mathrm{NaCl}, 0.01 \% \mathrm{SDS}$ or $0.05 \% \mathrm{H}_{2} \mathrm{O}_{2}$ at $25{ }^{\circ} \mathrm{C}$ for 3 days

the effect of sodium dodecyl sulfate (SDS) and $\mathrm{H}_{2} \mathrm{O}_{2}$ treatments. In the presence of $0.01 \%$ SDS or $0.05 \%$ $\mathrm{H}_{2} \mathrm{O}_{2}$, colony growth of the $\mathrm{FgHog} 1$ mutant was stunted but evident. In contrast, transformant $\mathrm{H}-4$ produced aerial hyphae (Fig. 2), similar to wild-type PH-1. These results indicated that expression of the MlpHogl gene in $F$. graminearum largely complemented the impaired response of the $\operatorname{Hog} 1$ mutant to cytoplasm membrane and oxidative stresses.

MlpHog1 partially restores sexual reproduction and plant infection defects in the F. graminearum Hogl mutant

Wild-type PH-1 formed numerous perithecia with cirrhi 2 weeks after self-fertilization on self-mating carrot agar, whereas the FgHogl mutants were sterile and could not produce perithecia (Fig. 3) (Zheng et al. 2012). Although transformant $\mathrm{H}-4$ did produce perithecia under the same conditions, the number of perithecia with cirrhi was reduced by approximately $40 \%$ (Fig. 3), suggesting that the sexual reproduction defect of the F. graminearum Hogl mutant was only partially complemented by the MlpHogl gene.
As FgHogl is important for plant infection (Zheng et al. 2012), we inoculated flowering wheat heads with wild-type $\mathrm{PH}-1, \mathrm{FgHog} 1$ mutant and transformant H-4 conidia suspensions to determine the virulence of the transformant. The wheat spikelets drop-inoculated with wild-type PH-1 developed typical wheat scab symptoms, whereas the FgHogl mutant was defective in spreading from the inoculated kernels to other spikelets on the same wheat head (Fig. 4) (Zheng et al. 2012). Conversely, transformant $\mathrm{H}-4$ did spread from the inoculated spikelet to neighbouring spikelets, but to a lesser extent than PH-1 (Fig. 4). At 14 dpi, the average disease index (diseased spikelets per head) was 6.67 for transformant $\mathrm{H}-4$ and 12.33 for PH-1 (Table 2). These results indicated that expression of the MlpHogl gene in F. graminearum partially complemented the plant infection defects exhibited by the Hogl mutant.

\section{Discussion}

Although Hog1 plays important roles during numerous biological processes, including osmoregulation, few studies have focused on its role in poplar foliar rust M. larici-populina. In this study, we cloned and characterized the Hog1 gene MlpHogl from M. laricipopulina. Based on genomic data provided by the completion of whole-genome sequencing projects, Hamel and colleagues analysed the structure of MAPK protein families in 24 fungal taxa, including both plant pathogens and mycorrhizal symbionts (Hamel et al. 2012). With regard to $M$. larici populina, they predicte that three MAPK systems are present in this fungus (Ste11Ste7-Kss1 pathway, Bck1-Mkk1-Slt2 pathway, and Ssk2-Pbs2-Hog1 pathway), including the intact Hog1 pathway. MlpHogl was cloned and sequenced from Chinese M. larici-populina strain Wh03 by homologybased cloning, harbouring a TGY phosphorylation site for the threonine/tyrosine phosphorylation required for kinase activation and a hallmark of stress-activated MAPKs in fungal and mammalian systems (Kultz 1998). Bioinformatic analysis revealed that its sequence shares high identity with Hogl othorlogs from other fungi. These results indicate the conservation of $\operatorname{Hog} 1$ across fungi and animals.

Heterologous expression is frequently used for the functional analysis of genes. For example, the yeast transformants expressing MoMsn 2 from M. oryzae restored growth to the $\Delta S c m s n 2$ yeast mutant on medium 
Fig. 3 Sexual reproduction of Fusarium graminearum strains. Self-crossing cultures of the wildtype strain (PH-1), FgHogl deletion mutant, and complemented strain $(\mathrm{H}-4)$ grown on carrot agar plates. The close-up views were obtained using a dissecting microscope
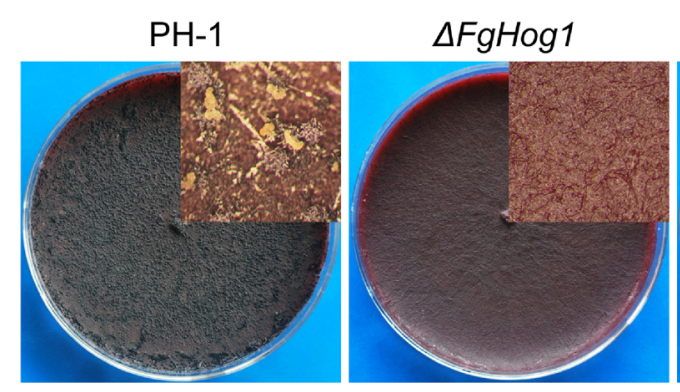

containing $5 \mathrm{mM} \mathrm{H} \mathrm{H}_{2}$ (Zhang et al. 2014). MAPK1 from the wheat stripe rust Puccinia striiformis partially complemented the corresponding kss 1 mutants of F. graminearum and M. oryzae (Guo et al. 2011). For the oomycete Phytophthora infestans, Picdc14 appears to be functionally equivalent to $c d c 14$ of $S$. cerevisiae based on its complementation of the $c d c 14-1^{\text {ts }}$ mutation (Ah Fong and Judelson 2003). In addition, Brassica oleracea $\mathrm{ABI} 5$ can rescue the ABA-insensitive phenotype of abi5-1 during seed germination (Zhou et al. 2013). Overall, the strictly biotrophic parasitism of rust fungi depend on the host's living tissue for their development, proliferation and reproduction (Feau et al. 2007), which has resulted in the lack of a stable transformation system for M. larici-populina. Therefore, we utilized a heterologous system to determine the potential function of genes of interest from M. larici-populina. Host-induced RNAi system is newly developed to indirectly suppress parasite gene expression by expressing an RNAi construct in vivo in the host (Nunes and Dean 2012). Recent studies show that this technology is also successfully used in obligate biotrophic fungi, such as Blumeria graminis and Puccinia striiformis f. sp. tritici

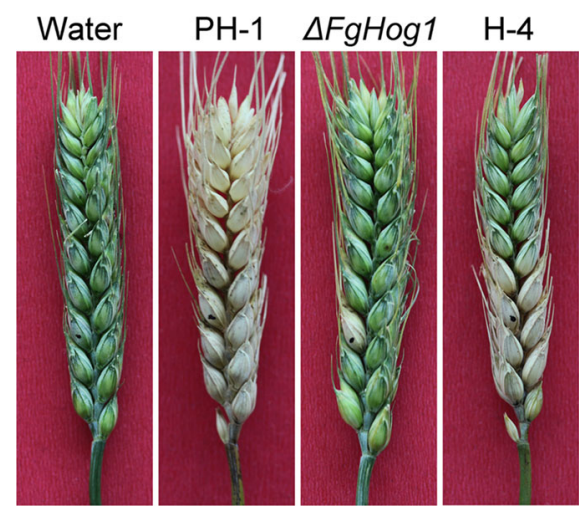

Fig. 4 Infection assays with flowering wheat heads. Wheat heads were drop-inoculated with sterile water or conidia from the wildtype strain (PH-1), FgHogl deletion mutant, and complemented strain (H-4). Typical heads were photographed at $14 \mathrm{dpi}$
(Nowara et al. 2010; Yin et al. 2011; Zhang et al. 2012). It may be available to study the parasite gene funtion for the poplar-M. larici-populina pathosystem in future.

The wild type FgHogl complements the defects of the Fghogl mutant (Zheng et al. 2012). Our observation showed that heterologous expression of MlpHog1 in FgHogl mutant did not fully complement the defects on environmental stresses, sexual reproduction and plant infection, which is similar to the phenomenon that PstMAPK1 and PstILC1 from Puccinia striiformis partially complemented the defects of corresponding ascomycetous mutants (Guo et al. 2011; Liu et al. 2014). Based on the bioinformatics analysis, the intact Hog1 pathway is present in M. larici-populina and MlpHog1 displays the high degree of conservation. However poplar foliar rust fungi have a distinct life style from $F$. graminearum, which may cause the differences on the regulation of biological processes including signal transduction between them. Thus rust genes including MlpHog1 are not fully functional in ascomycetous fungi.

Although the functions of Kss1/Fus3-type and Slt2type MAPKs in fungal pathogenesis appear to be broadly conserved, the role of Hogl homologues in pathogenesis differs drastically among plant pathogens (Zhao et al. 2007). M. oryzae Osm 1 is dispensable for plant infection (Dixon et al. 1999), as are Colletotrichum

Table 2 Disease index scores of Fusarium graminearum strains

\begin{tabular}{llll}
\hline & Strain & & \\
\cline { 2 - 4 } & PH-1 & $\Delta F g H o g 1$ & $\mathrm{H}-4$ \\
\hline Disease index $^{\mathrm{a}}$ & $12.33 \pm 2.89^{\mathrm{A}}$ & $1.00 \pm 0.00^{\mathrm{C}}$ & $6.67 \pm 0.58^{\mathrm{B}}$
\end{tabular}

\footnotetext{
${ }^{\text {a }}$ Diseased spikelets per wheat head were examined at $14 \mathrm{dpi}$. The mean and standard deviation were calculated from three independent experiments. The data from three independent replicates were analysed with Duncan's multiple range test. Different letters are used to show statistically significant differences $(P=0.05)$
} 
orbiculare Osc1 and Bipolaris oryzae Srm1 (Kojima et al. 2004; Moriwaki et al. 2006). In contrast, deletion of Mycosphaerella graminicola Hogl or B. cinerea Sakl produces nonpathogenic strains (Mehrabi et al. 2006; Segmuller et al. 2007). In the present study, we found that the complementated strains could spread from the inoculated spikelet to neighbouring spikelets, whereas the FgHogl mutant was defective in spreading under the same condition. Infection hyphae of M. laricipopulina ramify extensively and grow throughout the intercellular spaces of poplar leaves, producing haustoria for nutrient acquisition. Therefore, the partial complementation result with regard to plant infection indicates functional conservation between MlpHogl and FgHogl, and suggests that MlpHogl may play a role in regulating infectious growth in $M$. laricipopulina.

Although Hog1-type MAPKs play different roles in fungal virulence, their roles are conserved in the response to osmotic stress. Hog1 deletion mutants of B. cinerea, B. oryzae, C. orbiculare, C. parasitic and $M$. oryzae are all sensitive to hyperosmotic stress (Segmuller et al. 2007; Moriwaki et al. 2006; Kojima et al. 2004; Park et al. 2004; Dixon et al. 1999). In our study, MlpHog1 expression in F. graminearum partially complemented the hyperosmotic stress response defect of the FgHogl mutant, demonstrating functional conservation between MlpHogl and FgHogl. Moreover, the MlpHogl gene largely complemented the defective response to cytoplasm membrane and oxidative stresses in the FgHogl mutant. During the interaction between plants and pathogens, plants exert many kinds of stresses to pathogens, including osmotic stress, oxidative stress or cytoplasm membrane stress. Positive responses to these extracellular stresses may be beneficial for plant infection. These results indicate that $M l p H o g 1$ may play a role in responding to different environmental stresses as well as successful host infection.

Perithecium formation was not observed in the FgHogl mutant, however the complemented strains expressing the MlpHog1 gene could produce perithecia with cirrhi. Because of the decreased cirrhi formation efficiency, MlpHogl gene expression in $F$. graminearum partially complemented the defect in sexual reproduction, suggesting that $M l p H o g 1$ may be related to sexual reproduction. The vegetative cycle of M. larici-populina, which involves large amounts of dikaryotic urediniospores, occurs on poplar leaves, and successive cycles of uredinia formation throughout summer cause massive epidemics in poplar plantations throughout Europe and worldwide (Barres et al. 2012). Conversely, the sexual stage of this rust fungus occurs on larch needles (Larix sp.) in spring (Xhaard et al. 2011), persisting for a long time from the vegetative stage to the sexual stage. Additional work is required to explore the function of the MlpHogl gene during the sexual stage.

Acknowledgments This study was financially supported by the National Natural Science Foundation of China (No. 31300544). We thank Professor Jin-Rong Xu from NWAFU-PU Joint Research Center for providing the $F$. graminearum strains.

Open Access This article is distributed under the terms of the Creative Commons Attribution 4.0 International License (http:// creativecommons.org/licenses/by/4.0/), which permits unrestricted use, distribution, and reproduction in any medium, provided you give appropriate credit to the original author(s) and the source, provide a link to the Creative Commons license, and indicate if changes were made.

\section{References}

Ah Fong, A. M., \& Judelson, H. S. (2003). Cell cycle regulator Cdc14 is expressed during sporulation but not hyphal growth in the fungus-like oomycete Phytophthora infestans. Molecular Microbiology, 50(2), 487-494.

Barres, B., Dutech, C., Andrieux, A., Halkett, F., \& Frey, P. (2012). Exploring the role of asexual multiplication in poplar rust epidemics: impact on diversity and genetic structure. Molecular Ecology, 21(20), 4996-5008.

Bourett, T. M., Sweigard, J. A., Czymmek, K. J., Carroll, A., \& Howard, R. J. (2002). Reef coral fluorescent proteins for visualizing fungal pathogens. Fungal Genetics and Biology, 37(3), 211-220.

Bowden, R. L., \& Leslie, J. F. (1999). Sexual recombination in Gibberella zeae. Phytopathology, 89(2), 182-188.

Cao, Z., Li, Z., \& Hu, J. (1998). Physiological specialization in Melampsora larici-populina Kleb. in the Qinling mountains. Journal of Northwest Forestry College, 13(1), 53-57.

Cao, Z., Li, Z., \& Zhuang, J. (2000). Uredinales from the Qinling mountains. Mycosystema, 19(1), 13-23.

Chen, R. E., \& Thorner, J. (2007). Function and regulation in MAPK signaling pathways: lessons learned from the yeast Saccharomyces cerevisiae. Biochimica et Biophysica Acta, 1773(8), 1311-1340.

Cuomo, C. A., Guldener, U., Xu, J. R., Trail, F., Turgeon, B. G., Di Pietro, A., et al. (2007). The Fusarium graminearum genome reveals a link between localized polymorphism and pathogen specialization. Science, 317(5843), 1400-1402.

Ding, S., Mehrabi, R., Koten, C., Kang, Z., Wei, Y., Seong, K., et al. (2009). Transducin beta-like gene FTL1 is essential for pathogenesis in Fusarium graminearum. Eukaryotic Cell, $8(6), 867-876$. 
Dixon, K. P., Xu, J. R., Smirnoff, N., \& Talbot, N. J. (1999). Independent signaling pathways regulate cellular turgor during hyperosmotic stress and appressorium-mediated plant infection by Magnaporthe grisea. Plant Cell, 11(10), 20452058.

Duplessis, S., Major, I., Martin, F., \& Seguin, A. (2009). Poplar and pathogen interactions: insights from populus genomewide analyses of resistance and defense gene families and gene expression profiling. Critical Reviews in Plant Sciences, 28, 309-334.

Duplessis, S., Cuomo, C. A., Lin, Y. C., Aerts, A., Tisserant, E., Veneault-Fourrey, C., et al. (2011). Obligate biotrophy features unraveled by the genomic analysis of rust fungi. Proceedings of the National Academy of Sciences of the United States of America, 108(22), 9166-9171.

Feau, N., Joly, D. L., \& Hamelin, R. C. (2007). Poplar leaf rust: model pathogens for a model tree. Canadian Journal of Botany, 85, 1127-1135.

Gale, L. R., Chen, L. F., Hernick, C. A., Takamura, K., \& Kistler, H. C. (2002). Population analysis of Fusarium graminearum from wheat fields in Eastern China. Phytopathology, 92(12), $1315-1322$.

Guo, J., Dai, X., Xu, J. R., Wang, Y., Bai, P., Liu, F., et al. (2011). Molecular characterization of a Fus3/Kss 1 type MAPK from Puccinia striiformis f. sp. tritici, PsMAPK1. PloS One, 6(7), e21895.

Hacquard, S., Petre, B., Frey, P., Hecker, A., Rouhier, N., \& Duplessis, S. (2011). The poplar-poplar rust interaction: insights from genomics and transcriptomics. The Journal of Pathology, 2011, 716041.

Hamel, L. P., Nicole, M. C., Duplessis, S., \& Ellis, B. E. (2012). Mitogen-activated protein kinase signaling in plantinteracting fungi: distinct messages from conserved messengers. Plant Cell, 24(4), 1327-1351.

Herskowitz, I. (1995). MAP kinase pathways in yeast: for mating and more. Cell, 80(2), 187-197.

Hou, Z., Xue, C., Peng, Y., Katan, T., Kistler, H. C., \& Xu, J. R. (2002). A mitogen-activated protein kinase gene (MGV1) in Fusarium graminearum is required for female fertility, heterokaryon formation, and plant infection. Molecular Plant Microbe Interactions, 15(11), 1119-1127.

Kojima, K., Takano, Y., Yoshimi, A., Tanaka, C., Kikuchi, T., \& Okuno, T. (2004). Fungicide activity through activation of a fungal signalling pathway. Molecular Microbiology, 53(6), $1785-1796$.

Kultz, D. (1998). Phylogenetic and functional classification of mitogen- and stress-activated protein kinases. Journal of Molecular Evolution, 46(5), 571-588.

Liu, J., Wang, Q. L., Chang, Q., Han, L. N., Pei, G. L., Xue, Y. Q., et al. (2014). Isocitrate lyase is required for urediniospore germination of Puccinia striiformis f. sp. tritici. Molecular Biology Reports, 41(12), 7797-7806.

Mehrabi, R., Zwiers, L. H., de Waard, M. A., \& Kema, G. H. (2006). $M g H o g 1$ regulates dimorphism and pathogenicity in the fungal wheat pathogen Mycosphaerella graminicola. Molecular Plant - Microbe Interactions, 19(11), 1262-1269.

Monge, R. A., Roman, E., Nombela, C., \& Pla, J. (2006). The MAP kinase signal transduction network in Candida albicans. Microbiology, 152(Pt 4), 905-912.

Moriwaki, A., Kubo, E., Arase, S., \& Kihara, J. (2006). Disruption of SRM1, a mitogen-activated protein kinase gene, affects sensitivity to osmotic and ultraviolet stressors in the phytopathogenic fungus Bipolaris oryzae. FEMS Microbiology Letters, 257(2), 253-261.

Nowara, D., Gay, A., Lacomme, C., Shaw, J., Ridout, C., Douchkov, D., et al. (2010). HIGS: host-induced gene silencing in the obligate biotrophic fungal pathogen Blumeria graminis. Plant Cell, 22(9), 3130-3141.

Nunes, C. C., \& Dean, R. A. (2012). Host-induced gene silencing: a tool for understanding fungal host interaction and for developing novel disease control strategies. Molecular Plant Pathology, 13(5), 519-529.

Park, S. M., Choi, E. S., Kim, M. J., Cha, B. J., Yang, M. S., \& Kim, D. H. (2004). Characterization of HOG1 homologue, CpMK1, from Cryphonectria parasitica and evidence for hypovirus-mediated perturbation of its phosphorylation in response to hypertonic stress. Molecular Microbiology, 51(5), 1267-1277.

Robinson, M. J., \& Cobb, M. H. (1997). Mitogen-activated protein kinase pathways. Current Opinion in Cell Biology, 9(2), 180 186.

Segmuller, N., Ellendorf, U., Tudzynski, B., \& Tudzynski, P. (2007). BcSAK1, a stress-activated mitogen-activated protein kinase, is involved in vegetative differentiation and pathogenicity in Botrytis cinerea. Eukaryotic Cell, 6(2), 211-221.

Steenackers, J., Steenackers, M., Steenackers, V., \& Stevens, M. (1996). Poplar diseases, consequences on growth and wood quality. Biomass and Bioenergy, 10, 267-274.

Tian, C., Kang, Z., Li, Z. Q., Zhao, Y., \& Zhang, H. (2000). Analysis of the genetic specialization of Melampsora laricipopulina with RAPD markers. Scientia Silvae Sinica, 36(5), 54-58.

Treisman, R. (1996). Regulation of transcription by MAP kinase cascades. Current Opinion in Cell Biology, 8(2), 205-215.

Wan, Z., Li, Y., Chen, Y., Zhang, X., Guan, H., \& Yin, T. (2013). Melampsora larici-populina, the main rust pathogen, causes loss in biomass production of black cottonwood, plantations in the south of China. Phytoparasitica, 41, 337-344.

Wang, C., Zhang, S., Hou, R., Zhao, Z., Zheng, Q., Xu, Q., et al. (2011). Functional analysis of the kinome of the wheat scab fungus Fusarium graminearum. PLoS Pathogens, 7(12), e1002460.

Wang, G., Wang, C., Hou, R., Zhou, X., Li, G., Zhang, S., et al. (2012). The AMT1 arginine methyltransferase gene is important for plant infection and normal hyphal growth in Fusarium graminearum. PloS One, 7(5), e38324.

Widmann, C., Gibson, S., Jarpe, M. B., \& Johnson, G. L. (1999). Mitogen-activated protein kinase: conservation of a threekinase module from yeast to human. Physiological Reviews, 79(1), 143-180.

Xhaard, C., Fabre, B., Andrieux, A., Gladieux, P., Barres, B., Frey, P., et al. (2011). The genetic structure of the plant pathogenic fungus Melampsora larici-populina on its wild host is extensively impacted by host domestication. Molecular Ecology, 20(13), 2739-2755.

Yin, C., Jurgenson, J. E., \& Hulbert, S. H. (2011). Development of a host-induced RNAi system in the wheat stripe rust fungus Puccinia striiformis f. sp. tritici. Molecular Plant-Microbe Interactions, 24(5), 554-561.

Zhang, H., Guo, J., Voegele, R. T., Zhang, J., Duan, Y., Luo, H., et al. (2012). Functional characterization of calcineurin 
homologs PsCNA1/PsCNB1 in Puccinia striiformis f. sp. tritici using a host-induced RNAi system. PloS One, 7(11), e49262.

Zhang, H., Zhao, Q., Guo, X., Guo, M., Qi, Z., Tang, W., et al. (2014). Pleiotropic function of the putative zinc-finger protein MoMsn2 in Magnaporthe oryzae. Molecular Plant Microbe Interactions, 27(5), 446-460.

Zhao, X., Mehrabi, R., \& Xu, J. R. (2007). Mitogen-activated protein kinase pathways and fungal pathogenesis. Eukaryotic Cell, 6(10), 1701-1714.
Zheng, D., Zhang, S., Zhou, X., Wang, C., Xiang, P., Zheng, Q., et al. (2012). The FgHOGl pathway regulates hyphal growth, stress responses, and plant infection in Fusarium graminearum. PloS One, 7(11), e49495.

Zhou, X., Yuan, F., Wang, M., Guo, A., Zhang, Y., \& Xie, C. G. (2013). Molecular characterization of an ABA insensitive 5 orthologue in Brassica oleracea. Biochemical and Biophysical Research Communications, 430(3), 1140-1146. 